\title{
Wrong Potential Energy Term in Schrödinger's Equation for Hydrogen Atom
}

\author{
Gurcharn S. Sandhu \\ Independent Researcher, Chandigarh, India \\ Email: gurcharn.sandhu@gmail.com
}

How to cite this paper: Sandhu, G.S. (2018) Wrong Potential Energy Term in Schrödinger's Equation for Hydrogen Atom. Journal of Modern Physics, 9, 607-619. https://doi.org/10.4236/jmp.2018.94042

Received: February 15, 2018

Accepted: March 18, 2018

Published: March 21, 2018

Copyright $\odot 2018$ by author and Scientific Research Publishing Inc. This work is licensed under the Creative Commons Attribution International License (CC BY 4.0).

http://creativecommons.org/licenses/by/4.0/

\section{c) (i) Open Access}

\begin{abstract}
Even after nine decades of successful run of the Quantum Mechanics (QM), different viewpoints on foundational problems of Quantum Physics are still being actively debated. That is because mathematical logic of QM often defies the physical intuition which constitutes the main spirit of Physics. De Broglie's hypothesis of matter waves implied that the dynamic characteristics of a micro particle in motion, can be ascribed to the wave characteristics of the wavelet accompanying the particle. The Schrödinger equation models the matter-wave interactions through wavefunction $\psi$ and effectively serves as the foundation of QM. Even though mathematical structure of the Schrödinger equation is sound and elegant, here we show a conceptual mistake in the development of this equation wherein the physical situation has not been correctly modeled in the equation. The Coulomb potential energy of the proton electron pair in Hydrogen atom is essentially the negative interaction energy between their superposed electrostatic fields which is inversely proportional to their instantaneous separation distance. Assuming the proton to be relatively fixed at the origin of an appropriate coordinate system, the potential energy of the orbiting electron will be a function of instantaneous position coordinates of the electron. This has not been properly modeled in the Schrödinger equation. The resulting errors in the solution have been quantitatively demonstrated in this paper. We have stressed the necessity of incorporating a specific correction in the potential energy term of the Schrödinger equation, after which it may facilitate the adoption of Bohmian QM.
\end{abstract}

\section{Keywords}

Matter Waves, Potential Energy, Interaction Energy, Wavefunction, Electron Orbits

\section{Introduction}

As per De Broglie's hypothesis, all microscopic particles in motion are ac- 
companied by a wave-like propagation associated with the particle. This wave-phenomenon associated with the moving particle is characterized as matter-waves which are expected to be localized in the vicinity of the particle. De Broglie relations show that the wavelength $\lambda$ of the accompanying matter wave is inversely proportional to the momentum $p$ of the moving particle $(\lambda=h / p)$ and that the frequency $f$ is directly proportional to the particle's kinetic energy $T$. This implies that the dynamic characteristics of a micro particle in motion can be ascribed to the wave characteristics of the wavelet accompanying the particle. The intensity of the wavelet is expected to be large in the immediate vicinity of the particle and small elsewhere, implying thereby that the accompanying matter waves, even though extending into surrounding space, are nearly centered on the instantaneous location of the moving particle.

Schrödinger generalized the wave aspect of matter-waves by defining the wavefunction $\psi$ through the famous Schrödinger equation, which is a pure mathematical entity. The Schrödinger equation determines the evolution of wavefunction $\psi$ over time, and it is mathematically a type of wave equation. However, the wavefunction in quantum mechanics describes a kind of physical phenomenon, which is still open to different interpretations [1]. This wavefunction $\psi$ gives an appearance of wave behavior to matter, without demonstrating real physical waves propagating in the direction of motion of the particle [2]. However, in this wave picture of matter represented through the wavefunction $\psi$, the real physical particles got lost in the probabilities. This statistical picture of matter particles was never approved by de Broglie, who believed that the particle must move in the wave, in phase with it, and that it is wrong to consider a wave propagation without localization of the particle. Following the work of David Bohm, de Broglie continued to strive for a direct and real physical interpretation of matter-waves. The de Broglie-Bohm theory is today the only interpretation [3] giving real status to matter-waves in quantum theory.

The de Broglie-Bohm theory is characterized by the pilot-wave model and the causal interpretation of quantum mechanics. In Bohmian mechanics a matter particle in motion is described in part by its wave function, evolving according to Schrödinger's equation. Second part of the description specifies the actual positions of the particles through "guiding equation", which expresses the velocities of the particles in terms of the wavefunction. Thus, in Bohmian mechanics the configuration of a system of particles evolves via a deterministic motion choreographed by the wave function. However, the Bohmian mechanics has not been adopted in the mainstream and as per the Copenhagen interpretation of QM, the intensity of the wavelet is interpreted as the probability density for the location of the micro particle. That is, in QM, the location of the center of a micro particle in motion is assumed to be smeared across the whole region of the wavelet as position probability density.

It is significant to note that the proponent of matter-waves himself could not get convinced with their generalization through the wavefunction $\psi$ of Schrödinger equation. If the real moving particles get lost in the probability 
waves produced by the Schrödinger equation, there must be something seriously wrong either in the adaptation of original matter-wave concept or in the detailed development of the Schrödinger equation. It may be pointed out that Sommerfeld's model [4] of elliptical electron orbits, with quantized angular momentum and energy transitions, was a significant step forward from the Bohr model. When an electron moves in an elliptical orbit around the nucleus, its kinetic energy and potential energy both keep varying from instant to instant along the trajectory. However, Sommerfeld's model got scuttled with the development of Schrödinger equation. Here, we shall demonstrate a logical flaw in the development and interpretation of the Schrödinger equation. The assumption of time invariant potential energy term in the development of Schrödinger equation for Hydrogen atom was the first blunder which permanently restricted the electron motion along circular orbits.

\section{Schrödinger Equation for Free Particle}

The Schrödinger's wave equation may be considered as founded on L. de Broglie's suggestion of matter waves that accompany all micro particles in motion. These waves were assumed to represent the crucial dynamic characteristics of motion of the particle, namely the momentum $p$ and total energy $E$, through following two relations adapted from the photon wave packet.

$$
\begin{array}{r}
\boldsymbol{p}=h / \lambda \\
E=h v
\end{array}
$$

Here, $h$ is the Planck's constant, $\lambda$ the wavelength and $v$ the frequency of the motion induced waves accompanying the particle. Of course, unlike the photon wave packet, the motion induced wave packet accompanying a material particle is a separate entity-an appendage to the particle. Let us consider the motion of a free particle, say an electron.

Let point $A$ be the instantaneous center of an electron moving in free space with velocity $v$. This electron will be accompanied by a matter-wave field, spread around in the spatial vicinity of point $A$ as represented in Figure 1. Since the amplitude and intensity of the associated matter-waves are expected to be maximum at the particle location, we can expect the wave intensity to drop to zero at infinity or some large distance from the electron location $A$. For simplicity, we may demarcate the spatial boundary of the matter-wave field $S$ by assuming that the wave intensity at the boundary drops down to 0.01 percent (say) of the maximum value near the instantaneous electron location $A$. Let $Q$ be any space point (called field point) located within the boundary of matter-wave field region $S$ such that its distance from point $A$ may be represented by a vector $A Q=$ d.

Let this motion induced matter-wave packet $S$ be characterized by the parameter $\psi$ known as wave function. The $\psi$ is finite at all points within the region $S$ and effectively vanishes at the boundary of $S$. The whole region of space $S$ where the wave function $\psi$ is defined may be termed as the $\psi$ wave field. Since the 


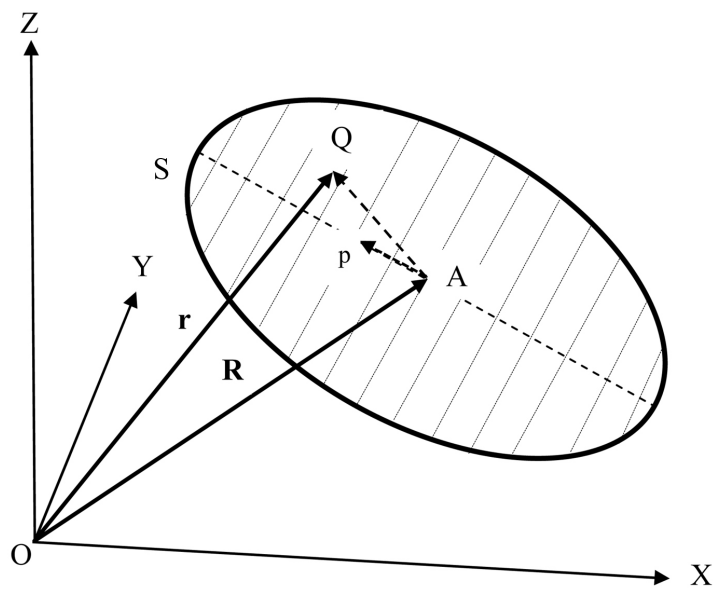

Figure 1. Representation of $\psi$ wave field S around an electron in motion.

electron location $A$ changes with time, the $\psi$ wave field will also change from instant to instant. As such the wave function $\psi$ at any field point $Q$ will depend on its distance vector $\boldsymbol{d}$ from the electron location $A$, as well as on time $t$. Hence the $\psi$ wave function at point $Q$ may be represented as $\psi(d, t)$. Let us now refer this $\psi$ wave field $S$ to an appropriate coordinate system $(X, Y, Z)$ centered at point $O$. Let $O A=R$ be the position vector of the instantaneous electron location $A$ and let $O Q=\boldsymbol{r}$ be the position vector of the field point $Q$. Therefore, the distance vector $\boldsymbol{d}$ can be expressed as,

$$
\boldsymbol{d}=\boldsymbol{r}-\boldsymbol{R}
$$

Accordingly, the $\psi$ wave function at point $Q$ may be represented as $\psi(\boldsymbol{r}, \boldsymbol{R}, t)$. Here, it needs to be emphasized that the wave function $\psi$, characterizing the wave field $S$, cannot be defined at or represented by the position coordinates of the point A alone, without accounting for the position coordinates of the general field point $Q$. That is, the wave function $\psi$ cannot be represented as $\psi(R, t)$ or $\psi(\boldsymbol{R})$ without taking into account the position vector $\boldsymbol{r}$ of the general field point $Q$. The position vector $R$ of the electron location point A is governed by the electron trajectory equation,

$$
\boldsymbol{R}=f(t)
$$

Substituting for $R$ from the trajectory equation into the wave function, the $\psi$ wave function at point $Q$ may get transformed to $\psi(\boldsymbol{r}, t)$. The $\psi(\boldsymbol{r}, t)$ in general will be a complex function of space and time coordinates. The intensity of the wave function will be given by $|\psi|^{2}$ which is also known as the probability density. This wave function $\psi(r, t)$ is obtained as a solution of Schrödinger's wave equation. The Schrödinger's equation in turn is derived from the energy conservation principle as applied to the moving particle, by making use of the following two operators, which form the core of Quantum Mechanics.

$$
\begin{gathered}
\boldsymbol{p} \rightarrow-\imath \hbar \nabla \\
E \rightarrow \imath \hbar \partial . / \partial t
\end{gathered}
$$


where iota $\iota=$ square root of $(-1)$ and $\hbar=h / 2 \pi$. Expressing kinetic energy $T$ in terms of momentum $p$ and mass $m$ of the electron in motion, we have,

$$
E=T=p^{2} / 2 m
$$

Multiplying Equation (7) with $\psi(r, t)$ and applying the operators (5) and (6), we get,

$$
i \hbar \frac{\partial \psi(r, t)}{\partial t}=-\left(\frac{\hbar^{2}}{2 m}\right) \cdot \nabla^{2} \psi(r, t)
$$

This is the well-known Schrödinger's wave equation for a free particle [5].

\section{Notions of Kinetic, Potential and Total Energy}

\subsection{Kinetic Energy and the $\psi$ Wave Field}

If the kinetic energy of a free particle is reduced to zero, we can see from Equations (5) to (8) that $\nabla \psi$ and $\partial \psi / \partial t$ will reduce to zero. That is, in the absence of kinetic energy, the $\psi$ wave field of the particle will collapse to zero. This shows a direct correlation between the kinetic energy of a particle in motion and the existence of the $\psi$ wave field. Since the energy density in electrostatic and magnetic fields is proportional to the squares of electric and magnetic field strengths, it is quite possible that the kinetic energy of the moving particle may be existing as field energy of the associated $\psi$ wave field. According to one estimate [6] about 35 percent of the rest mass of the electron is contained in its electrostatic field energy. In contrast to the current interpretation, the intensity of the wave function or $|\psi|^{2}$, may actually represent the kinetic energy density of the particle. As such, difference in dynamic or relativistic [7] mass and the rest mass of an electron in motion may be contained in the field energy of its $\psi$ wave field. We can generally say that any change in the motion of a particle will induce a corresponding change in the kinetic energy as well as the overall $\psi$ wave field of that particle and vice versa.

\subsection{Potential Energy as Interaction Energy}

Let us consider the case of an isolated electron. The electrostatic field of an electron, with radially decaying electric field strength, can be identified with its field energy density proportional to the square of the electric field strength. A significant portion of the mass energy of the electron is actually stored or contained in this electrostatic field. The field energy component of the electron mass is an integral part of the electron and is not dependent on the existence of any other charge or field in its vicinity. Now, let us consider a proton and electron pair separated by distance $R$. Their respective electrostatic fields will get superposed almost throughout their spatial extension. Consequently, the combined field energy of the proton-electron system, being proportional to the square of the resultant field strength, will be slightly less than the total sum of the individual field energies of the isolated charges. This reduction in the combined field energy of the proton-electron system is precisely the negative interaction energy due 
to the Coulomb interaction and is known as the negative potential energy of the proton electron pair. Therefore,

Potential energy of proton-electron pair $=V=-e^{2} / 4 \pi \varepsilon_{0} R$

Or, interaction energy released by the system $=|V|=e^{2} / 4 \pi \varepsilon_{0} R$

The energy released from the proton-electron field interaction, as given by Equation (10), is converted into the kinetic energy of the electron, assuming the proton to be relatively at rest. As the kinetic energy of a particle is contained in its $\psi$ wave field, potential energy of the proton-electron pair may signify the transfer of interaction energy released from the combined electrostatic field of the system to the $\psi$ wave field of the electron. Thus, a +ve potential energy of a particle signifies the transfer of $\psi$ wave field energy (i.e. K.E.) to the field energy (i.e. mass energy) of the interacting particles. Similarly, a -ve potential energy signifies the transfer of a part of the combined field energy to the $\psi$ wave field energy of the interacting particles. The Schrödinger's wave equation is intended to describe the variations in $\psi$ wave field of a moving particle as a result of such energy transfers.

It is therefore obvious that the potential energy of an electron with respect to a proton at distance $R$, represented as $V(R)$, cannot be regarded as a field parameter in the sense that it does not represent any entity distributed in space. For example, the electrostatic field or field energy density can be regarded as field parameters because they represent the entities which are existing or defined at all space points of the associated field at any instant of time. On the other hand, potential energy is the interaction energy depending entirely on relative location of the electron with respect to the proton at any particular instant and is not defined or existing at any other space point at that instant.

If an electron is totally isolated or infinitely separated from all other charged particles, then it will experience no electrostatic interaction with any other particle and will be effectively at zero potential. Therefore, the term "potential energy" is not applicable for a single isolated particle due to absence of any interaction. It has a meaning only for two or more interacting particles or fields, wherein transfer of energy could take place between the combined electrostatic field energy and the $\psi$ wave field energies of the interacting particles. If at any instant $t$, the proton (considered almost stationary) is located at point $\mathrm{O}$, the origin of coordinate system (Figure 1) and the moving electron is located at point A with position vector $R$, then the potential energy of the electron will depend on the magnitude of $R$ and represented by $V(R)$. It will not be a function of the coordinates of field point $Q(r)$ that defines the $\psi$ wave field. That is, when the wave function is represented as $\psi(r, t)$ to characterize the matter-wave field of the electron with instantaneous position vector $R$, the potential energy term cannot be represented as $V(r)$ in place of correct representation $V(R)$.

\subsection{Total Energy as Externally Supplied or Removed Energy}

The total energy $E$ of a system of two interacting particles (the proton-electron 
pair in the present case) is intended to represent the sum total of mass energies, including electrostatic field energies, plus any external energy added or subtracted from the system. In actual practice however, the mass energies of the interacting particles are regarded as invariable constant and removed from consideration. Therefore, the total energy $E$ of a system is assumed to be zero when the particles are infinitely separated. When the particles approach one another to a separation distance $R$ and their fields get superposed, their potential energy and kinetic energy still sum up to zero if no external energy is supplied or removed from the interacting system. In all other cases, when some finite energy content is either added to or removed from the system, sum total of the potential and kinetic energies is a finite number which is called the total energy of the interacting system.

$$
E=K . E .+P . E .=T+V(R)
$$

Total energy $E$ is +ve when this amount of energy is externally added or supplied to the system of interacting particles and is -ve when it is extracted, or taken out of the system. The externally supplied energy may either get added to the kinetic energy of the system or to their electrostatic field energies through the potential energy term and vice versa. Generally, a negative $E$ will represent a bound state of the system of interacting particles and therefore the total energy $E$ is also called binding energy of the system. When this binding energy or the so called total energy $E$ gets removed or emitted out of the system, this energy is ultimately extracted from the mass energies of the interacting particles. Even though a constant total energy $E$ or a stationary energy state implies the constancy of sum of K.E. and potential energy of the system, there could still be tremendous energy exchange oscillations between the kinetic and potential energies of the interacting particles.

\section{Schrödinger's Equation with Wrong Potential Energy Term}

With the inclusion of potential energy term in Equation (7), the total energy $E$ is given by Equation (11) which may be written in terms of momentum $p$ as,

$$
E=\frac{p^{2}}{2 m}+V(R)=\frac{p^{2}}{2 m}+\frac{e^{2}}{4 \pi \epsilon_{0} R}
$$

Accordingly, Equation (8) gets modified to,

$$
\imath \hbar \frac{\partial \psi(r, t)}{\partial t}=-\left(\frac{\hbar^{2}}{2 m}\right) \cdot \nabla^{2} \psi(r, t)+V(R) \psi(r, t)
$$

The complex relationship between the variations of total, kinetic and potential energy, and the corresponding space-time variations of the $\psi$ wave field representing the kinetic energy, is reflected through the Schrödinger's wave Equation (13) involving the potential energy term $V(R)$. However, the standard Schrödinger's wave equation is normally written in the form, 


$$
\imath \hbar \frac{\partial \psi(r, t)}{\partial t}=-\left(\frac{\hbar^{2}}{2 m}\right) \cdot \nabla^{2} \psi(r, t)+V(r) \psi(r, t)
$$

Equations (13) and (14) differ in the P.E. terms $V(R)$ and $V(r)$.

In the Schrödinger's original wave Equation (14), the potential energy is expressed as a function of the coordinates of general field point $Q(\boldsymbol{r})$, instead of the coordinates of instantaneous location $A(R)$ of the particle (Figure 1). That means the Schrödinger's wave Equation (14) is founded on the total energy relation,

$$
E=T+V(r)=\frac{p^{2}}{2 m}+\frac{e^{2}}{4 \pi \epsilon_{0} r}
$$

instead of Equation (12). As already discussed above, the potential energy of an electron-proton pair is strictly a function of their instantaneous relative distance $R$ and is not defined at any other space point $Q(\boldsymbol{r})$. This discrepancy is not a simple or inadvertent mistake in the Schrödinger's wave Equation (14) but rather a serious conceptual mistake with far reaching consequences. This mistake is continued with throughout Quantum Mechanics, where the potential energy term $V(r)$ is often replaced by $e . \phi(r)$; with scalar potential $\phi(r)$ treated as a function of coordinates of general field point $Q(\boldsymbol{r})$ rather than a function of coordinates of instantaneous location $A(R)$ of the particle. The greatest temptation for permitting this mistake might have been the consequent ease of solving the Schrödinger's Equation (14) by treating the potential energy term $V(r)$ as spherically symmetric and independent of time. Even though most weaknesses of Quantum Mechanics could be attributed to this conceptual mistake, yet for want of timely rectification, the mistake had to be "swept under the probability carpet".

\section{Consequential Wrong Solutions of Schrödinger's Equation}

Let us now examine a few consequential wrong solutions of Schrödinger's wave Equation (14), arising out of the above-mentioned mistake in the potential energy term $V(r)$. For this, let us consider the ground state 1s orbital solution of Hydrogen atom, the normalized wave function $\psi_{n l m}=\psi_{100}(r, t)$ of which is,

$$
\psi_{100}(r, t)=\sqrt{\left(\frac{1}{\pi a_{0}^{3}}\right)} \cdot \mathrm{e}^{-r / a_{0}} \cdot \mathrm{e}^{-i E_{1} t / \hbar}
$$

where, $E_{1}=2.18 \times 10^{-18} \mathrm{~J}=-13.6 \mathrm{eV}$ is the total energy of the $1 \mathrm{~s}$ orbital and $a_{0}=$ $0.053 \mathrm{~nm}$ is the Bohr radius.

\subsection{Spherically Symmetric and Oscillating $\psi$ Wave Field}

In accordance with the original suggestion of L. de Broglie, the solution for ground state 1s orbital of Hydrogen was expected to yield some sort of waves or wave packet, accompanying the orbiting electron. But Equation (16) represents spherically symmetric standing wave oscillations of the $\psi$ wave field, which does 
not correspond to the physical situation. Equation (16) cannot represent any traveling wave or a wave group which could describe the orbiting motion of the electron. Hence, fundamentally this solution is unsuitable to represent the physical situation and should have been rejected. This error could be attributed to the wrong potential energy term $V(r)$ used in the Schrödinger's Equation (14), as discussed above.

At any instant the intensity of $\psi$ wave function is expected to be maximum in the vicinity of location of the electron at that instant. But from Equation (16) we get,

$$
P(r)=\left|\psi_{100}\right|^{2}=\left(\frac{1}{\pi a_{0}^{3}}\right) \mathrm{e}^{-2 r / a_{0}}
$$

Equation (17) shows that the intensity of the $\psi$ wave function is spherically symmetric with its maximum value at the center where the proton is located. The obvious conclusion from this result could be that the electron and proton are both located at the center, which of course is physically impossible. The physical situation demanded that the $\psi$ wave packet should not only have accompanied the orbiting electron but also should have been centered at and spread around the instantaneous location of the electron.

Therefore, it could be concluded that solution (16) does not represent the physical situation and should have been rejected as invalid. This error too could be attributed to the wrong potential energy term $V(r)$ used in the Schrödinger's Equation (14). Therefore, the Schrödinger's Equation (14) needs to be corrected with the potential energy term $V(R)$ and should be solved in accordance with Bohmian mechanics [8], with pre-set initial and boundary conditions to yield a complete solution consisting of,

1) The electron trajectory $R=f(t)$;

2) The wave function $\psi(\boldsymbol{r}, \boldsymbol{R}, t)$ or $\psi(\boldsymbol{r}, t)$.

\subsection{Implied Negative Kinetic Energy of the Electron}

As per the usual terminology in QM, Equation (16) represents the $\psi$ wave function for the lowest stationary state of electron in Hydrogen atom. For this stationary state, the probability density $P(r)$ is given by Equation (17). The integral of this probability density over the entire $\psi$ field (i.e. for $r$ varying from zero to infinity) works out to unity, as expected, since $\psi$ is normalized. This result is interpreted as the overall probability of finding the electron within the entire $\psi$ field is $100 \%$. Let us now work out the overall probability of finding the electron within a spherical shell of inner radius $R_{1}=0.5 a_{0}$ and outer radius $R_{2}=1.5 a_{0}$ where $a_{0}$ is the Bohr radius.

$$
P_{12}=\int_{R_{1}}^{R_{2}} P(r) 4 \pi r^{2} \mathrm{~d} r=0.4965 \text { by using } P(r) \text { from Equation (17) }
$$

That means the overall probability of finding the electron within a spherical shell of radii $0.5 a_{0}$ and $1.5 a_{0}$ is $49.65 \%$. Apparently, this is quite a reasonable result. Now let us carry out one more computation. This time let us work out the 
overall probability of finding the electron outside a sphere of radius $R_{3}=2 a_{0}$. Proceeding on the same lines as above, we get this probability as,

$$
P_{3}=\int_{R_{3}}^{\infty} P(r) 4 \pi r^{2} \mathrm{~d} r=\frac{4}{a_{0}^{3}} \int_{2 a_{0}}^{\infty} \mathrm{e}^{-2 r / a_{0}} \cdot r^{2} \mathrm{~d} r=0.2381
$$

That means the overall probability of finding the electron outside a sphere of radius $2 a_{0}$ is about 23.81 percent. A closer look will show that this result is totally wrong. Since the result (16) is valid in the region outside a sphere of radius $2 a_{0}$, the Equations (14) and (15) must also be valid. Therefore, for constant total energy $E_{1}=-13.6 \mathrm{eV}=-2.176 \times 10^{-18} \mathrm{~J}$, from Equation (15) we get,

$$
T=E_{1}-\left[-\frac{e^{2}}{4 \pi \epsilon_{0} r}\right]=\frac{e^{2}}{4 \pi \epsilon_{0} r}-2.176 \times 10^{-18} \text { Joules }
$$

This shows that kinetic energy $T$ of the electron keeps reducing with increasing $r$. Equation (18) shows that $T$ reduces to zero at $r=2 a_{0}$. That means when the electron is located outside a sphere of radius $2 a_{0}$, its kinetic energy will become negative. But we have seen above that probability of finding the electron outside a sphere of radius $2 a_{0}$ is 23.81 percent. Hence, we draw the conclusion that as per original Schrödinger's Equation (14), there is $23.81 \%$ probability that the electron, in ground state of Hydrogen atom, will exist in a negative kinetic energy state with imaginary velocity components. Since this is patently an absurd conclusion, we must review the situation. Therefore, we come back to our previous observation that the potential energy term $V$ in the original Schrödinger's Equation (14) has been wrongly taken as a function of coordinates of general field point $Q(r)$, instead of taking it as a function of coordinates of point $A(R)$, the instantaneous location of the electron.

\section{Sommerfeld Model Type Elliptical Electron Orbit for $1 \mathrm{~s}$ Hydrogen Atom}

We have seen in the previous section that a typical solution (Equation (16)) for the ground state 1s of Hydrogen atom, obtained from the original Schrödinger's Equation (14), is physically untenable and wrong. In comparison, let us glance through a corresponding solution for an elliptical electron orbit for $1 \mathrm{~s}$ Hydrogen atom obtained with Sommerfeld model [9] approach. A computed elliptical orbit for the $1 \mathrm{~s}$ electron is shown in Figure 2. Radial distance of the electron from the nucleus varies from about $0.13 a_{0}$ to $1.87 a_{0}$ over the complete orbit.

Two main inputs for this electron trajectory model are the discrete total energy $E$ levels and the quantized angular momentum $L$ of the orbiting electron. Since the total energy is just the energy removed or emitted out of the system and it is known from the Planck relation that the energy of the emitted photons is quantized in terms of $h v$ or $\hbar \omega$, the discrete total energy $E$ can thus be correlated with principal quantum number $n$. Further, since the emission of a photon of energy $\hbar \omega$ corresponds to a change in electron angular momentum $\Delta L$ by one $\hbar$, quantization of electron angular momentum as $k \hbar$ can thus be understood. 


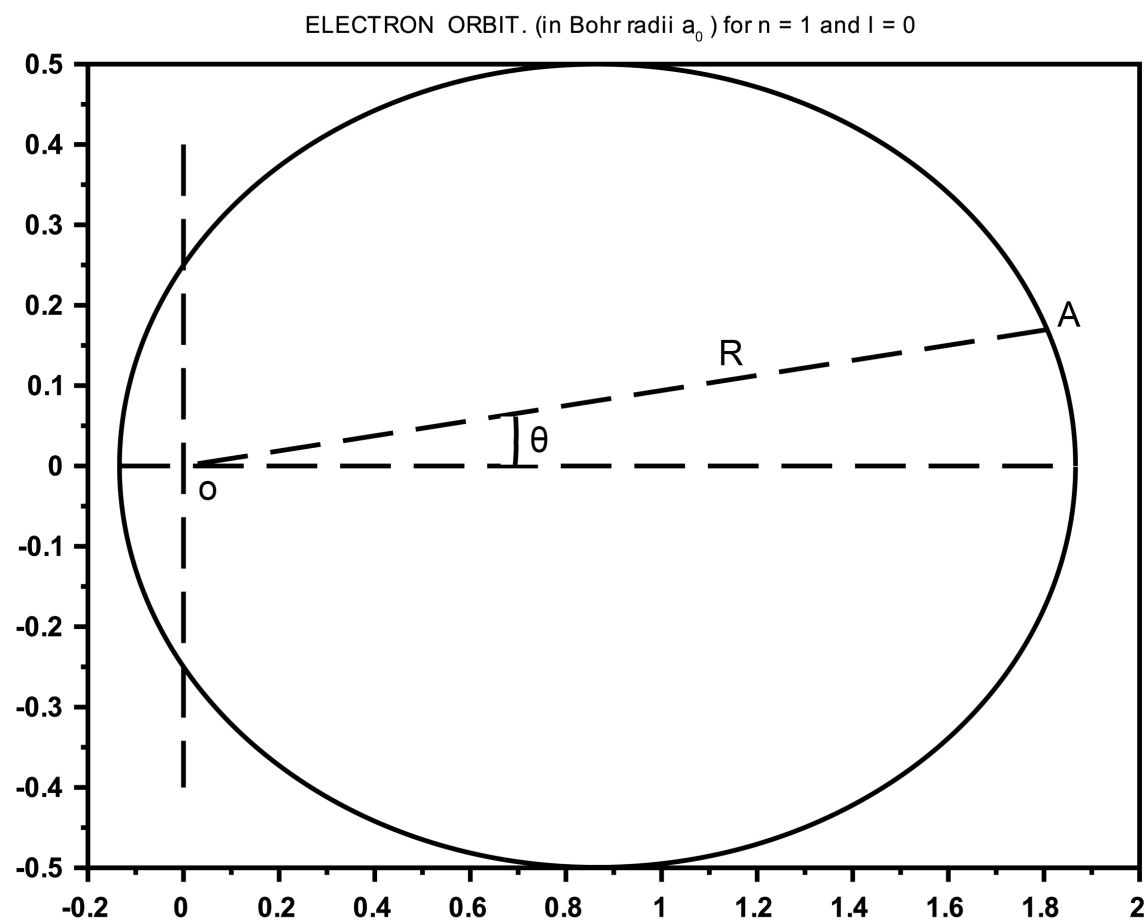

Figure 2. Computed elliptical orbit for 1s electron in Hydrogen atom.

However, in our model [10] to ensure $\Delta L=\hbar$, we have correlated the parameter $k$ with the usual quantum number $l$ as $k=l+1 / 2$ instead of $\sqrt{l(l+1)}$.

As shown in Figure 3, the linear acceleration of the orbiting electron can be of the order of $10^{24} \mathrm{~m} / \mathrm{s}^{2}$. When orbiting electron experiences such high accelerations, its accompanying de Broglie waves are expected to get compressed to form a sort of transient shock front.

Since for a hydrogen atom, an ionization energy of $13.6 \mathrm{eV}\left(2.18 \times 10^{-18} \mathrm{~J}\right)$ is required to force the electron from its lowest energy level entirely out of the atom [11], the $1 \mathrm{~s}$ electron in Hydrogen atom is identified with $-13.6 \mathrm{eV}$ total energy. However, Figure 4 shows that the kinetic energy of the orbiting electron varies from a minimum of about one $\mathrm{eV}$ to a maximum of about $190 \mathrm{eV}$. This fluctuation in kinetic energy is also accompanied by a corresponding fluctuation in potential energy $V(R)$ of the orbiting electron. This much fluctuation in the potential energy $V(R)$ occurs in just about $1.5 \times 10^{-16}$ seconds time period. That is why the assumption of time invariant potential energy term in original Schrödinger's Equation (14) is wrong.

\section{Concluding Remarks}

In spite of the error in potential energy term $V$, as brought out above, the QM has been quite useful in the study of high energy micro particle interactions. For those applications where either the effect of potential energy term is negligible or the potential energy function $V$ is made more dependent on particle locations, the QM has been of immense value. However, the solutions of Schrödinger's 


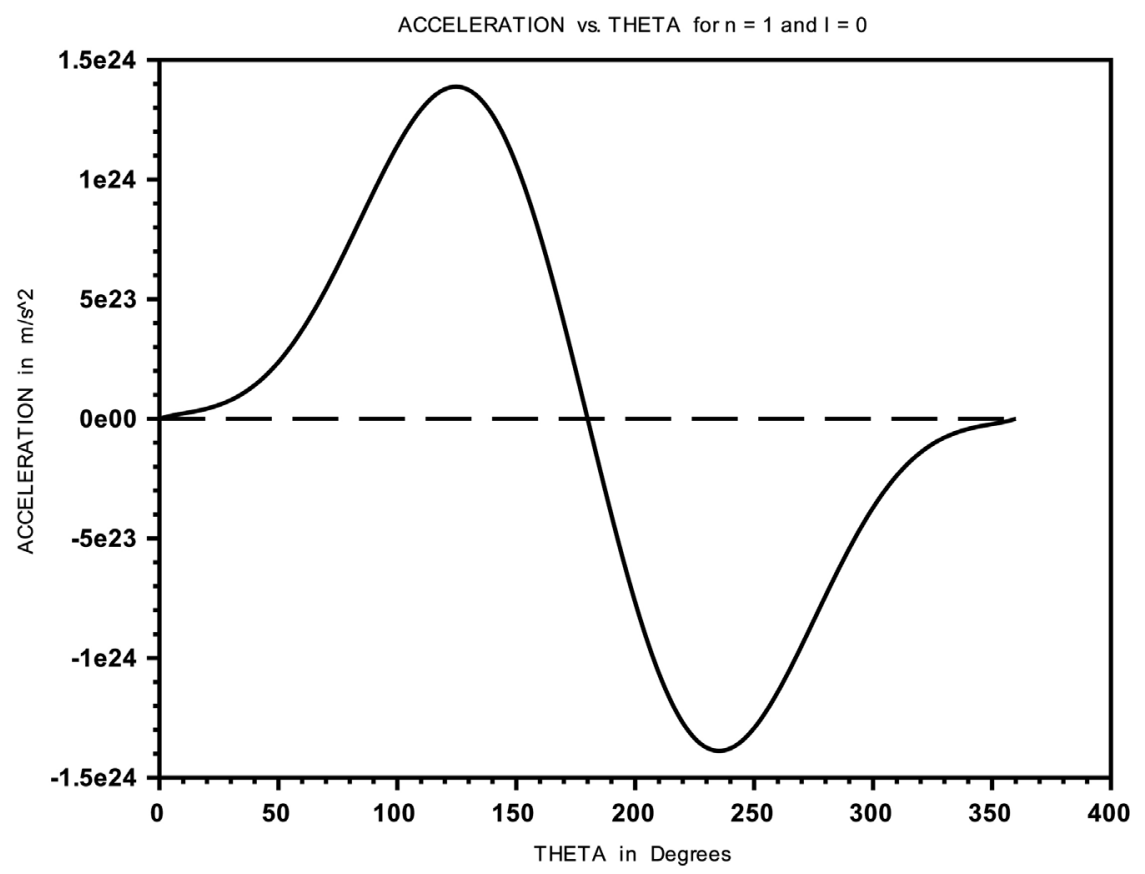

Figure 3. Linear acceleration profile for $1 \mathrm{~s}$ electron in hydrogen atom.

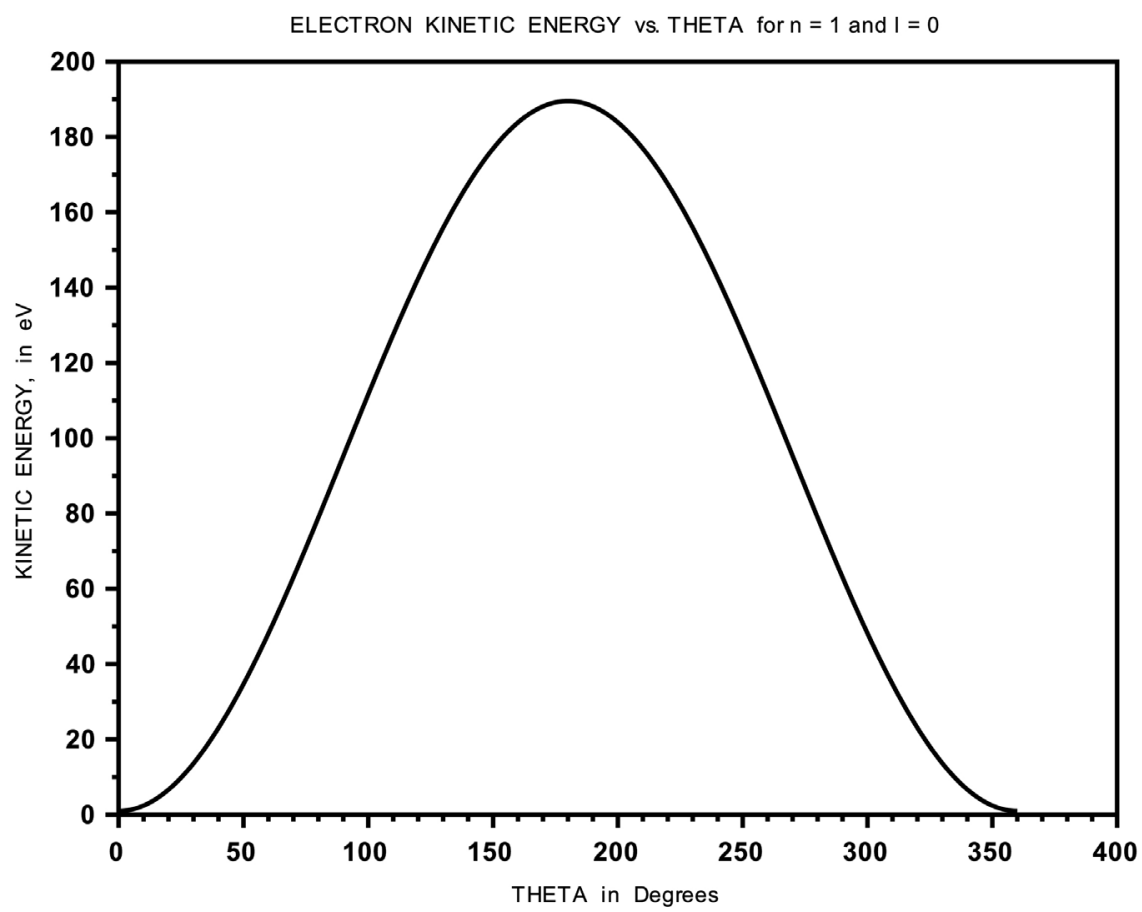

Figure 4. Electron kinetic energy profile for $1 \mathrm{~s}$ electron in hydrogen atom.

equation for different energy states of electron in Hydrogen atom appear to describe only the time averaged charge density distributions around nucleus and not the trajectories of electrons. That is because the potential energy term $V$ in the equation has been assumed as time invariant and not dependent on the instantaneous position coordinates of the electron. Since the position coordinates 
of the electron have been inadvertently omitted in the input to the equation, naturally the exact position of the electron is lost in the final solution. It is hoped that through rectification of the error in potential energy term $V$ in the Schrödinger's wave equation, we may be in a better position to further enhance the efficacy and utility of QM, possibly by adopting the methodology and the spirit of Bohmian Mechanics.

\section{References}

[1] Khrennikov, A., et al. (2015) Foundations of Physics, 2, 1-4.

[2] Khrennikov, A. (2017) Foundations of Physics, 47, 1077-1099. https://doi.org/10.1007/s10701-017-0089-0

[3] Bohm, D. (1952) Physical Review, 85, 166-193.

[4] Eckert, M. (2014) The European Physical Journal H, 39, 141-156. https://doi.org/10.1140/epjh/e2013-40052-4

[5] Ward, D.W., et al. (2006) How to Derive the Schrodinger Equation. arXiv:physics/0610121v1 [physics.hist-ph]

[6] Sandhu, G.S. (2009) Fundamental Nature of Matter and Fields. iUniverse Inc., Bloomington, IN, 33.

http://bookstore.iuniverse.com/Products/SKU-000127260/Fundamental-Nature-ofMatterand-Fields.aspx

[7] Sandhu, G.S. (2016) Applied Physics Research, 8, 45.

[8] Oriols, X., et al. (2012) Overview of Bohmian Mechanics. arXiv:1206.1084 [quant-ph]

[9] Deeney, T., et al. (2014) American Journal of Physics, 82, 883. https://doi.org/10.1119/1.4881955

[10] Sandhu, G.S. (2017) Applied Physics Research, 9, 44.

[11] The Editors. Encyclopedia Britannica. Ionization Energy. https://www.britannica.com/science/ionization-energy 